(CONOR). We calculated the difference between the observed number of hip fractures in 2019 and the expected number of hip fractures given stable fracture rates. IFPPs in 2019 attributable to changes in prevalences of $\mathrm{BMI}>25$ were estimated according to sex and 5-year age groups from 50 to $85+$.

Results Hip fracture rates decreased by 28\% from 1999 to 2019 with 2,549 fewer hip fractures observed than expected in 2019. The prevalences of BMI > 25 had increased in all age groups in both men and women over the time period. Unadjusted estimates showed that the increased prevalences of BMI $>25$ accounted for $\sim 30 \%$ of the decline. Increased BMI explained $\sim 50 \%$ of the total number of IFFPs in men and $\sim 25 \%$ of the total IFPPs in women.

Conclusion The incident hip fracture rates in Norway declined between 1999 and 2019. These preliminary results suggest that increased BMI in the population, reflecting a shift in the population BMI distribution, has significantly contributed to the declining hip fracture incidence, particularly in men.

\section{OP21 COMPARING ASSOCIATIONS OF TOTAL AND CENTRAL OBESITY WITH INCIDENT ARTHRITIS: RESULTS FROM THE ENGLISH LONGITUDINAL STUDY OF AGEING}

${ }^{1}$ Rozemarijn Witkam*, ${ }^{1}$ James Gwinnutt, ${ }^{1,2}$ Jennifer Humphreys, ${ }^{3}$ Rachel Cooper ${ }^{1}$ David Selby, ${ }^{1,2}$ Suzanne Verstappen. ${ }^{1}$ Centre for Epidemiology Versus Arthritis, The University of Manchester, Manchester, UK; ${ }^{2}$ NIHR Manchester Biomedical Research Centre, The University of Manchester, Manchester, UK; ${ }^{3}$ Department of Sport and Exercise Sciences, Manchester Metropolitan University, Manchester, UK

\subsection{6/jech-2021-SSMabstracts.21}

Background Body mass index (BMI) and waist circumference (WC) are the measures most commonly used to identify total and central obesity, respectively. Research on the associations between obesity and incident rheumatoid arthritis (RA) and osteoarthritis (OA) is limited, particularly for central obesity. Therefore, we investigated the associations between obesity defined by both BMI and WC and incident RA and OA in England.

Methods The English Longitudinal Study of Ageing (ELSA) is a nationally representative panel study of non-institutionalised adults aged $\geq 50$ years, with biannual waves of data collection (2002-2019). Participants with at least one nurse visit measuring height, weight and WC and one follow-up assessment were included in this study. BMI of $\geq 30 \mathrm{~kg} / \mathrm{m}^{2}$ and $\mathrm{WC} \geq 102$ $\mathrm{cm}$ for men and $\geq 88 \mathrm{~cm}$ for women defined total and central obesity, respectively. Outcomes were self-reported incident RA and OA during follow-up. Prevalent RA or OA cases at baseline were excluded. Cox proportional hazards models were used to investigate the associations between total and central obesity and incident RA and OA separately, controlling for baseline covariates (i.e. age, gender, ethnicity, education, occupation, wealth, smoking and alcohol consumption). Gender differences were formally tested by including interaction terms between gender and obesity. Analyses were conducted using Stata v14.

Results The RA and OA analyses included 10,931 (54.1\% female; mean age 64.0 (standard deviation (SD) 9.6); mean follow-up 8.8 (SD 4.2) years; and 1,216 incident cases of RA) and 9,281 (51.3\% female; mean age 63.6 (SD 9.6); mean follow-up 7.8 (SD 4.2) years; and 2,369 incident cases of OA) participants, respectively. In both samples, more women than men had total and central obesity. Total and central obesity were both associated with incident RA and these associations were maintained after adjustment for covariates (fully-adjusted hazard ratios (HRs) 1.58 (95\% confidence interval (CI) 1.39, $1.80)$ and 1.43 (95\% CI 1.25, 1.62), respectively). Similarly, total and central obesity were both associated with incident OA (fully-adjusted HRs 1.45 (95\% CI 1.32, 1.60) and 1.42 (95\% CI 1.30, 1.55), respectively). There was no evidence of gender differences in any of these associations ( $\mathrm{p}$-values from tests of interaction $>0.33$ ).

Conclusion Both total and central obesity were associated with increased rates of RA and OA among adults aged 50 years and older. There were no gender differences for these associations. Education about obesity and the development of both $\mathrm{RA}$ and OA may result in better prevention or early intervention strategies.

\section{OP22 THE GLOBAL PREVALENCE OF FEMALE GENITAL MUTILATION: A SYSTEMATIC REVIEW AND META- ANALYSIS OF NATIONALLY REPRESENTATIVE STUDIES}

${ }^{1}$ Leen Farouki, ${ }^{1}$ Zeinab El Dirani, ${ }^{2}$ Sawsan Abdulrahim, ${ }^{1}$ Christelle Akl, ${ }^{1}$ Chaza Akik, ${ }^{1}$ Stephen J McCall*. 'Center for Research on Population and Health, American University of Beirut, Beirut, Lebanon; '2Department of Health Promotion and Community Health, American University of Beirut, Beirut, Lebanon

\subsection{6/jech-2021-SSMabstracts.22}

Background Female Genital Mutilation (FGM) entails the removal, cutting and modification of the external female genitalia for non-medical reasons. The UN Sustainable Development Goal 5 (SDG 5.3) on gender equality calls for ending all traditional harmful practices, including FGM by 2030. This systematic review examined the prevalence of FGM and its subtypes globally, by WHO region, and by country.

Methods A systematic search using $\mathrm{MeSH}$ headings and keywords from inception to March 2, 2020 was undertaken in MEDLINE, PsycINFO, Web of Science, and EMBASE to identify studies that presented data on FGM prevalence. Only nationally representative studies were included in the meta-analysis. Abstract and full-text screening, quality assessment, and data extraction were undertaken independently by two reviewers. Pooled FGM prevalence was estimated by meta-analysis using a Freeman-Tukey double arcsine transformation and a random effects model using $\mathrm{R}$ software. FGM prevalence and types of FGM were presented separately by women and girls. Sub-group analysis was presented by WHO region.

Results Out of 3205 articles identified in the search, 28 nationally representative studies were included in the metaanalysis, and these studies included estimates for women and girls in 27 and 34 countries, respectively. The pooled prevalence estimate of FGM in women aged $15-49$ was $40 \%$ (95\% CI:26-55\%; $\left.\mathrm{I}^{2}=100 \%\right)$ and 15\% (95\% CI:10-21\%; $\left.\mathrm{I}^{2}=100 \%\right)$ in girls aged $0-14$ years old. The country with the highest FGM prevalence in women was Guinea (97\%) and the lowest, Uganda $(0.3 \%)$. The highest prevalence in girls was in Mali (77\%), and the lowest in Ghana (0\%). The most common FGM type was having flesh removed, at $69 \%$ (95\% CI:59-79\%) of women and 70\% (95\% CI: 55-84\%) of girls, followed by having the genital area sewn shut with $11 \%$ of women (95\% CI:4-20\%) and $9 \%$ of girls $(95 \%$ CI:6-12\%). The pooled prevalence by WHO region was 39\% (95\% CI: 24-56\%) for women and 15\% (95\% CI:9$22 \%)$ for girls in Africa, and 52\% (95\% CI:8-93\%) for 
women and 20\% (95\% CI:6-39\%) for girls in the Eastern Mediterranean.

Conclusion There is large global variation in FGM, with the Eastern Mediterranean region recording the highest prevalence. Reassuringly, FGM is lower among girls, highlighting a declining trend in the practice. Some included reports may have underestimated FGM prevalence in girls due to continued risk; future studies should use age cohort analyses to monitor decline. Policy and community-level interventions are needed to meet SDG target 5.3.

\section{OP23 PARENTAL CONSENT FOR TIME-CRITICAL NEONATAL TRIALS IN LOW AND MIDDLE-INCOME COUNTRIES: IS IT TRULY INFORMED?}

${ }^{1}$ Stuti Pant*, ${ }^{2}$ Maya Annie Elias, ${ }^{3}$ Kerry Woolfall, ${ }^{1}$ Sudhin Thayyil. ${ }^{1}$ Centre for Perinatal Neuroscience, Imperial College London, London, UK; ${ }^{2}$ Perinatal Trials Unit Foundation, Bangalore, India; ${ }^{3}$ Institute of Population Health, University of Liverpool, Liverpool, UK

\subsection{6/jech-2021-SSMabstracts.23}

Background Parental consent rates for neonatal interventional trials are significantly higher in Low and middle-income countries (LMIC) than in high-income countries, raising concerns about the credibility of the consent processes (Patterson et al PLOS One 2021). We conducted a mixed-methods study to understand the informed consent process in a neonatal cooling trial [Hypothermia for encephalopathy in low and middleincome countries (HELIX) trial] conducted in India, Sri Lanka and Bangladesh.

Methods Term infants with neonatal encephalopathy, aged less than six hours were randomly allocated to cooling therapy or usual care, following informed parental consent. The consenting process was audio-video $(\mathrm{A}-\mathrm{V})$ recorded in all cases. We analysed the A-V records of the consent process using a 5point Likert scale on three parameters - Empathy, Information, Autonomy. Additionally, we used exploratory observation method to capture relevant aspects of consent process and discussions between parents and professionals. Finally, we conducted in-depth interviews with a subgroup of 20 parents and 15 health care professionals. A thematic analysis was performed on the observations of $\mathrm{A}-\mathrm{V}$ records and on the interview transcripts.

Results In HELIX trial, a total of 475 parents were approached, of which $408(86 \%)$ consented. Of these, 294 $\mathrm{A}-\mathrm{V}$ records were analysed. Median (Interquartile range) score for empathy, information, autonomy was 5 (0), 5 (1) and 5 (1) respectively. However, thematic analysis suggested that the parental decision to participate was based on a unreserved trust in the treating doctors, therapeutic misconception, and access to an expensive treatment free of cost. Most parents did not understand the concept of a clinical trial, nor the nature of the intervention. Lower levels of parental education and misinformation further convoluted the voluntary informed consent process. Parents were visibly incapacitated, and many told the doctor to do whatever is best for the baby. Clinicians lacked equipoise and were biased towards cooling therapy as it was already a standard of care in high-income countries, and this influenced parental decision making. However, the HELIX trial results subsequently showed cooling was harmful and increased mortality in these settings.

Conclusion Despite rigorous research governance and consent process, parental decisions were heavily influenced by situational incapacity and a trust in doctors to make the right decision on their behalf. Further research is required to identify culturally and context appropriate strategies to ensure truly informed trial participation.

\section{OP24 HEALTH IMPACTS, SURVIVAL, QUALITY-ADJUSTED LIFE YEARS, AND COSTS OF CHRONIC KIDNEY DISEASE IN CHILEAN ADULTS}

${ }^{1}$ Magdalena Walbaum*, 'Shaun Scholes, ${ }^{2}$ Ruben Rojas, 'Jennifer Mindell, ${ }^{3}$ Elena Pizzo. ${ }^{1}$ Research Department of Epidemiology and Public Health, University College London, London, UK; ${ }^{2}$ School of Health and Related Research, University of Sheffield, UKi ${ }^{3}$ Department of Applied Health Research, University College London, London, UK

\subsection{6/jech-2021-SSMabstracts.24}

Background Chronic Kidney Disease (CKD), a leading public health problem, poses substantial burdens for both healthcare systems and patients. The aim of this study was to estimate the health and economic burden of CKD for adults aged $40+$ years from the perspective of the Chilean public healthcare system, by adapting the Schlackow and colleagues' CKD-cardiovascular disease (CVD) model.

Methods The microsimulation CKD-CVD model was built based on the Study of Heart and Renal Protection (SHARP). We adapted it using Chilean data, and combining two submodels. The CKD submodel included five mutually exclusive states replicating CKD progression based on the Kidney Disease: Improving Global Outcomes (KDIGO) classification: from CKD stage $3 b$ to end-stage kidney disease (ESKD). The CVD submodel used the individuals' annual risks of cardiovascular outcomes (both fatal and non-fatal) and non-vascular death. Both submodels were combined into a first-order Markov model with annual cycles to allow the inclusion of all the possible states between the CKD stages and the CVD outcomes. We used nationally-representative Chilean survey and registry data to adapt the model with a time horizon of 20 years, from 2020 to 2040. Costs, life-years (Lys), and qualityadjusted life-years (QALYs) were discounted using a 3\% discount rate after the first year.

Results On average, an individual aged 64 years, starting with CKD stage $3 \mathrm{~b}$ at baseline, had a projected cumulative probability of progressing to ESKD of 0.16 (95\% credibility interval CI: $0.13-0.20)$; and projected survival of 11.9 years $(95 \% \mathrm{CI}$ : 11.2-12.5) and 8.9 QALYs (95\% CI: 8.3-9.5). An individual starting in ESKD and having renal replacement therapy had projected survival of 7.7 years (95\% CI: 7.0-8.4) and 5.7 QALYs (95\% CI: 5.1-6.2). For individuals in CKD Stage 3b, the predicted probabilities of having a major vascular event (MVE) or vascular death (VD) were 0.14 (0.11-0.18), 0.26 $(0.21-0.33)$ and $0.43(0.35-0.54)$ in the next 5, 10 and 20 years, respectively. For individuals in ESKD, the predicted probabilities of having a MVE or VD over the same time periods were $0.31(0.26-0.37), 0.46(0.39-0.53)$ and 0.59 (0.51-0.68); mean lifetime direct healthcare costs were $£ 70,606$ (95\% CI: 57,385-85,190).

Conclusion The important interdependence between CKD and CVD outcomes and the lower life expectancy and quality-adjusted survival when individuals progress to more advanced stages of the disease highlight the need for effective public health policies to address the management of the disease and its risk factors at primary and secondary healthcare level. 